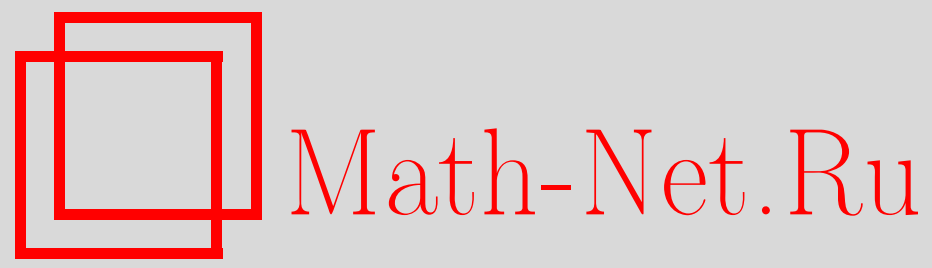

И. М. Дектярев, Равнораспределенность дивизоров для последовательностей голоморфных кривых, Функи. анализ и его прил., 2000, том 34, выпуск $3,17-25$

DOI: https://doi.org/10.4213/faa308

Использование Общероссийского математического портала MathNet.Ru подразумевает, что вы прочитали и согласны с пользовательским соглашением

http: //www . mathnet.ru/rus/agreement

Параметры загрузки:

IP : 54.174 .149 .18

26 апреля 2023 г., 03:04:12

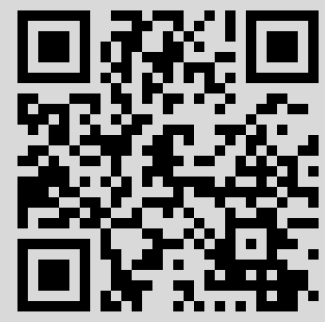


Функциональньй анализ и его приложения

2000, т. 34, вып. 3, с. 17-25

УДК 515.16

\title{
Равнораспределенность дивизоров для последовательностей голоморфных кривых
}

\author{
(c) 2000. И. М. ДЕКТяРев
}

Многомерная теория распределения значений очень приблизительно может быть описана следующим образом. Имеется голоморфное отображение некомпактного комплексного многообразия, на котором задана функция исчерпания, в компактное. Задача состоит в изучении функций, связывающих характеристики «роста» отображения многообразия с характеристиками «роста» прообразов точек или дивизоров. Изучается также структура множества исключительных (дефектных) значений или дивизоров, т.е. таких значений или дивизоров, для которых поведение указанных функций отличается от «типичного». При этом «рост» соотносится со значениями функции исчерпания.

В последнее время появился ряд работ, в частности, связанных с комплексной динамикой, в которых подобные вопросы изучаются для последовательностей отображений (в основном, полиномиальных). При этом роль функции исчерпания играет степень полиномов, задающих отображение. Первой работой в этом направлении, по-видимому, является статья [7]. К этому же направлению относится и настоящая работа.

Мы будем изучать голоморфные кривые $f: \mathbb{C} \rightarrow Q$ в (не обязательно компактном) $n$-мерном комплексном многообразии $Q$, на котором задано семейство дивизоров, параметризованное $m$-мерным $(m>1)$ компактным комплексным многообразием $M$. Обозначая через $D_{y}$ дивизор в $Q$, соответствующий значению $y \in M$, рассмотрим в прямом произведении $Q \times M$ множество $W=$ $\left\{(q, y): q \in D_{y}\right\}$. Всюду в дальнейшем мы будем предполагать, что $W$ является подмногообразием и что проекция $\theta: W \rightarrow Q$ является регулярным сюръективным, а проекция $\pi: W \rightarrow M$ - открытым отображениями. Проекцию $\theta$ мы будем рассматривать как расслоение с базой $Q$ и слоем $S_{q}=\left\{y: y \in M, q \in D_{y}\right\}$ над точкой $q$.

Будем, далее, предполагать, что на многообразии $M$ задана такая эрмитова метрика, что ассоциированная с ней $(1,1)$-форма $\lambda$ удовлетворяет тождеству $d d^{c}\left(\lambda^{m-1}\right) \equiv 0$, а форма объема $\omega=\lambda^{m} / m !-$ условию $\int_{M} \omega=1$. Существование таких метрик доказано в работе [1]. Там же доказано, что на $M$ существует такое семейство положительных функций $\gamma_{a}(y)=\gamma(y, a)$, зависящее от параметра $a \in M$, что выполнены следующие условия:

(a) при $y \neq a$ функция $\gamma_{a}(y)$ является гладкой по совокупности переменных;

(b) при $y \neq a$ имеет место соотношение $d d^{c}\left(\gamma_{a}(y) \mu\right)=\omega$, где форма $\mu$ - это $(m-1)$-я внешняя степень формы $\lambda$;

*Работа выполнена при поддержке Российского фонда фундаментальных исследований, грант 98-01-00867. 
(с) в окрестности точки $a$ функция $\gamma_{a}(y)$ имеет разложение

$$
\gamma_{a}(y)=\frac{1}{4(m-1) \pi^{m}} \rho(a, y)^{2-2 m}+O\left(\rho^{3-2 m}\right),
$$

где $\rho(a, y)$ - расстояние между точками $a$ и $y$ относительно используемой эрмитовой метрики, а $O$-оценка равномерна по всему $M$.

Через $\psi$ обозначим форму на $Q$, получающуюся из $\pi^{*} \omega$ послойным интегрированием по слоям расслоения $\theta$, а через $\zeta_{a}(q)$ - функцию на $Q$, задаваемую формулой $\zeta_{a}(q)=\int_{S_{q}} \gamma_{a}(y) \mu$.

ПримеР 1. Пусть $Q$ и $M$ - взаимно двойственные $n$-мерные проективные пространства, получившиеся в результате проективизации унитарных пространств с заданными взаимно сопряженными базисами. Координаты в этих базисах обозначим соответственно через $\left(q_{0}, q_{1}, \ldots, q_{n}\right)$ и $\left(y_{0}, y_{1}, \ldots, y_{n}\right)$ и зададим $W$ формулой

$$
W=\{(q, y): q \in Q, y \in M,\langle q, y\rangle=0\} .
$$

Форма $\lambda$ на $M$ - это $(1,1)$-форма, ассоциированная со стандартной метрикой Фубини-Штуди и соответствующим образом нормированная. В карте, определяемой условием $y_{0}=1$, она задается формулой $\lambda=(4 \pi)^{-1} \sqrt[n]{n !} d d^{c} \ln (1+$ $\left.\left|y_{1}\right|^{2}+\cdots+\left|y_{n}\right|^{2}\right)$, а функция $\gamma_{a}(y)$ задается формулой

$$
\gamma_{a}(y)=\frac{1}{4 \pi(n !)^{(n-1) / n}}\left(\ln \frac{1}{\sin ^{2} \alpha}+\sum_{1}^{n-1} \frac{1}{k} C_{n-1}^{k} \operatorname{tg}^{-2 k} \alpha\right),
$$

где через $\alpha$ обозначен модуль угла между векторами, задающими точки $a$ и $y$.

Форма $\psi$, будучи инвариантной относительно унитарных преобразований, получается из $(1,1)$-формы, ассоциированной со стандартной метрикой Фубини-Штуди на $Q$, умножением на константу. Чтобы вычислить эту константу, выберем карты на $Q$ и на $M$, задав их условиями $q_{1}=-1$ и $y_{0}=1$. Рассмотрим значение в точке $(0,-1,0, \ldots, 0)$ ограничения формы $\psi$ на одномерное множество $R=\left\{\left(q_{0},-1,0, \ldots, 0\right)\right\}$. В качестве локальных координат на $\theta^{-1}(R)$ выберем $\left(q_{0}, y_{2}, \ldots, y_{n}\right)$. Проекция $\pi$ при этом задается формулой $y_{1}=q_{0}$, а форма $\pi^{*} \omega$ - выражением $\left((4 \pi)^{-1} d d^{c} \ln \left(1+\left|q_{0}\right|^{2}+\left|y_{2}\right|^{2}+\cdots+\left|y_{n}\right|^{2}\right)\right)^{n}$. Таким образом, коэффициент формы $\psi$ при $d q_{0} \wedge \overline{d q_{0}}$ в заданной точке равен

$$
\frac{n !}{\pi^{n}} \frac{i}{2} \int_{\mathbb{C}^{n-1}} \frac{d V_{\text {евкл }}^{2 n-2}}{\left(1+\left|y_{2}\right|^{2}+\cdots+\left|y_{n}\right|^{2}\right)^{n+1}}=\frac{i}{2 \pi} .
$$

Итак, $\psi=(4 \pi)^{-1} d d^{c} \ln \left(1+\left|q_{0}\right|^{2}+\left|q_{2}\right|^{2}+\cdots+\left|q_{n}\right|^{2}\right)$ (в карте, определяемой равенством $\left.q_{1}=-1\right)$.

Функция $\zeta_{a}(q)$ в описанных выше координатах задается формулой $\zeta_{a}(q)=$ $(4 \pi)^{-1} \ln \left(\langle a, a\rangle\langle q, q\rangle /|\langle a, q\rangle|^{2}\right)$.

На комплексной прямой $\mathbb{C}$ зададим форму $\eta=(2 \pi)^{-1}\left(1+|z|^{2}\right)^{-2} d z \wedge d \bar{z}$ и рассмотрим пространство $\Phi$ дважды непрерывно дифференцируемых финитных функций, на котором определим норму функции $\varphi$ как точную нижнюю грань таких положительных $\nu$, что формы $\nu \eta+d d^{c} \varphi$ и $\nu \eta-d d^{c} \varphi$ положительны. Всюду в дальнейшем мы будем рассматривать только невырожденные кривые $f: \mathbb{C} \rightarrow Q$ (т. е. такие кривые, что образ не содержится целиком ни в 
одном дивизоре). Для невырожденной кривой построим функционалы на пространстве $\Phi$, являющиеся в определенном смысле аналогами соответствующих величин теории Неванлинны. (В классическом случае голоморфных кривых при подходящем выборе пробных функций значения этих функционалов после несложных преобразований сводятся к этим величинам.)

Определим на пространстве $\Phi$ функционал $N(f, a)$ как сумму значений функции $\varphi$ на прообразах точек пересечения рассматриваемой кривой с дивизором $D_{a}$, подсчитанную с учетом кратности. Функционал $T(f)$ определим как интеграл по $\mathbb{C}$ от формы $\varphi f^{*} \psi$, а функционал $m(f, a)$ - как интеграл по $\mathbb{C}$ от формы $\zeta_{a}(f(z)) d d^{c} \varphi$.

Имеет место следующий аналог первой основной теоремы теории Неванлинны.

ТЕоремА 1. Для любой функиии $\varphi \in \Phi$ июбого значения $a \in M$ выполняется соотношение $T(f) \varphi=N(f, a) \varphi+m(f, a) \varphi ;$ при этом функииональи $N(f, a)$ и $m(f, a)$ слабо непрерьвно зависят от $a$.

ДокАзАтельство. Кривая $f: \mathbb{C} \rightarrow Q$ порождает индуцированное расслоение $\Theta=f^{*} \theta$. Через $X$ обозначим пространство этого расслоения $\{(x, y): x \in \mathbb{C}$, $\left.y \in M, f(x) \in D_{y}\right\}$, а через $F$ - отображение $X \rightarrow M$, задаваемое формулой $F(x, y)=y$.

Из невырожденности кривой $f: \mathbb{C} \rightarrow Q$ следует, что отображение $F$ является открытым. Действительно, рассматривая это отображение локально и выбрав подходящие координаты, будем считать, что кривая $f$ задается набором таких функций $\left\{f_{1}, \ldots, f_{n}\right\}$, что $f_{1}(0)=0, \ldots, f_{n}(0)=0$. Будем также считать, что в окрестности точки $\left\{q_{1}=0, \ldots, q_{n}=0, y_{1}=0, \ldots, y_{m}=0\right\}$ многообразие $W$ задается уравнением $G\left(q_{1}, \ldots, q_{n}, y_{1}, \ldots, y_{m}\right)=0$. Функция $G\left(f_{1}(z), \ldots, f_{n}(z)\right.$, $0, \ldots, 0)$, обращаясь в нуль при $z=0$, не может быть равна нулю тождественно, иначе образ кривой целиком содержался бы в дивизоре, соответствующем точке из $M$ с координатами $(0, \ldots, 0)$. Таким образом, мы можем к функции $G\left(f_{1}(z), \ldots, f_{n}(z), y_{1}, \ldots, y_{m}\right)$ применить подготовительную лемму Вейерштрасса, откуда открытость отображения $F$ видна непосредственно.

Обозначая теперь через $\varphi_{1}$ функцию $\Theta^{*} \varphi$, легко убедиться, что $T(f) \varphi=$ $\int_{X} \varphi_{1} \omega$. Далее, $N(f, a) \varphi=\sum \varphi_{1}\left(x_{i}\right)$, где суммирование (с учетом кратности) распространяется на все те $x_{i} \in X$, для которых $F\left(x_{i}\right)=a$. Наконец, $m(f, a) \varphi=$ $\int_{X} d d^{c} \varphi_{1} \wedge F^{*}\left(\gamma_{a} \mu\right)$. Воспользуемся буквами $T, N$ и $m$ для обозначения соответствующих величин, связанных с отображением $F$. Итак, нам осталось доказать, что $T(F) \varphi_{1}=N(F, a) \varphi_{1}+m(F, a) \varphi_{1}$ и что функционалы в правой части слабо непрерывно зависят от $a$. Но именно это составляет содержание теоремы 1 работы [2]. Там эта теорема доказывалась в несколько иных условиях, однако при доказательстве использовались только те свойства отображения и форм, которые имеются и в рассматриваемом случае.

СледСтвиЕ 1. Относительно введенной на Ф нормь норма функиионала $T(f)-N(f, a)$ при любом значении а не превосходит величиньл $\int_{\mathbb{C}} \zeta_{a}(f(z)) \eta$, которую мь будем обозначать $m_{\eta}(f, a)$.

В дальнейшем мы будем рассматривать только кривые, для которых значение интеграла $\int_{\mathbb{C}} f^{*} \psi$ конечно, и будем называть это значение степенью кривой. (В условиях рассмотренного выше примера, если отображение $f$ в однородных координатах на $Q$ задается системой полиномов, не имеющих общих корней, 
этот интеграл равен максимальной из степеней полиномов, задающих отображение. См., например, [5, с. 188].)

ОПредЕЛЕНиЕ 1 . Пусть $f_{1}, \ldots, f_{k}, \ldots$ - последовательность таких кривых и $d_{1}, \ldots, d_{k}, \ldots$ - последовательность их степеней. Через $\delta_{N}(a)$ обозначим величину $\liminf _{k \rightarrow \infty} m_{\eta}\left(f_{k}, a\right) / d_{k}$ и назовем ее неванлинновским дефектом дивизора, соответствующего значению $а$ в пространстве параметров. Аналогично определим валироновский дефект $\delta_{V}(a)$ как $\lim \sup _{k \rightarrow \infty} m_{\eta}\left(f_{k}, a\right) / d_{k}$. Дивизоры, дефект которых отличен от нуля, будем называть дефектнылми.

Используя функцию $\gamma_{a}$ в качестве ядра, можно построить на пространстве $M$ теорию потенциала. Характер особенностей этой функции таков, что получающиеся свойства во многом аналогичны свойствам ньютоновского потенциала. В частности [6, с. 253], если емкость какого-либо множества $B$ равна $\mathrm{Cap}(B)$, то для любого $\delta>0$ найдется такое покрытие этого множества шарами радиусов $r_{1}, r_{2}, \ldots$, что $\sum_{k} r_{k}^{2 m-2+\delta}<L \operatorname{Cap}(B)^{1+\delta /(2 m-2)}$, где константа $L$ зависит только от размерности.

Теорема 2. Пусть компактное множество $B_{0} \subset M$ coстоит из таких точек а, что норма функиионала $T(f)-N(f, a)$ для этих а больше, чем $A$. Тогда емкость $\operatorname{Cap}\left(B_{0}\right)$ этого множества не превосходит величинь $t / A$, где через $t$ обозначено максимальное значение функиии $\int_{S_{q}} \mu$ на $Q$. (Если заданная на $М$ эрмитова метрика является кэлеровой, то эта функиия есть константа. В частности, для рассмотренного выше примера эта константа равна $(n !)^{(n-1) / n}$.) Эта же оченка верна и для множества B тех точек, для которых вьгполнено неравенство $m_{\eta}(f, a)=\int_{\mathbb{C}} f^{*} \zeta_{a} \eta>A$.

ДокАЗАтельство. Следствие 1 утверждает, что $B_{0} \subset B$, и потому достаточно доказать только второе утверждение. Проинтегрируем неравенство, задающее множество $B$, по равновесной мере на этом множестве, воспользуемся определением функции $\zeta_{a}$ и поменяем порядок интегрирования. Мы получим, что $A \operatorname{Cap}(B)$ не превосходит величины

$$
\int_{M}\left(\int_{\mathbb{C}}\left(\int_{S_{f(z)}} \gamma_{a}(y) \mu\right) \eta\right) \sigma(d a)=\int_{\mathbb{C}}\left(\int_{S_{f(z)}}\left(\int_{M} \gamma_{a}(y) \sigma(d a)\right) \mu\right) \eta,
$$

откуда следует нужная оценка.

ТЕоремА 3. Если последовательность кривьх такова, ито для некоторой положительной константь $\alpha$ их степени удовлетворяют соотношению $d_{k}>$ $\alpha k$, то множество $\Delta$ значений параметров, соответствующих дивизорам, дефектным по Валирону (и, тем более, дефектныл в смисле Неванлинньи), имеет хаусдорфову размерность, не большую чем $2 m-2$.

ДокАЗАтЕЛьство. Зададимся положительными $\varepsilon$ и $\delta$ и покажем, что множество $\Delta$ можно покрыть системой шаров, радиусы $r_{i}$ которых удовлетворяют неравенству $\sum r_{i}^{2 m-2+\delta}<\varepsilon$. Построим для каждого $k$ множество $B_{k}$ тех значений $a \in M$, для которых выполнено неравенство $d_{k}^{-1} \int_{\mathbb{C}} \zeta_{a}\left(f_{k}(z)\right) \eta>d_{k}^{-\delta /(3 m)}$. Eмкость множества $B_{k}$, согласно теореме 2 , не превосходит величины $t d_{k}^{-1+\delta /(3 m)}$, и, значит, его можно покрыть системой шаров, сумма радиусов которых не превосходит величины $L \operatorname{Cap}\left(B_{k}\right)^{1+\delta /(2 m-2)} \leqslant L_{1} d_{k}^{-1-\delta /(2 m(2 m-1))}$. С другой стороны, из условия на последовательность кривых следует, что каждое $a$, соответствующее дефектному дивизору, содержится во всех множествах из некоторой 
бесконечной подпоследовательности последовательности $B_{k}$, т. е. для любого $s$ выполнено соотношение $\Delta \subset \bigcup_{k>s} B_{k}$. Выбрав $s$ так, чтобы выполнялось неравенство $L_{1} \sum_{k>s} d_{k}^{-1-\delta /(2 m(2 m-1))}<\varepsilon$, мы получим нужное утверждение. Для кривых в проективном пространстве подобный результат другими методами был получен в работе [7].

Полученную оценку размерности множества дефектных дивизоров, как это видно из приводимых ниже примеров, в общем случае улучшить нельзя.

ПримеР 2. Пусть в условиях примера 1 кривая $f_{k}$ задается системой функций $\left\{1, z, \ldots, z^{n-1}, z^{k}\right\}$. Мы будем рассматривать дивизоры, соответствующие значениям $a$, у которых последняя координата равна нулю, т.е. дивизоры, проходящие через точку $q_{0}$ с однородными координатами $(0, \ldots, 0,1)$. Интеграл $\int_{\mathbb{C}} f_{k}^{*} \zeta_{a} \eta$ в этом случае представляется в виде суммы не зависящего от $k$ интеграла $(4 \pi)^{-1} \int_{\mathbb{C}} \eta \ln \left(\langle a, a\rangle /\left|\left\langle a, f_{k}(z)\right\rangle\right|^{2}\right)$ и интеграла $(4 \pi)^{-1} \int_{\mathbb{C}} \eta \ln \left(|z|^{2 k}+\right.$ $\left.\sum_{i=0}^{n-1}|z|^{2 i}\right)$. Выражение, стоящее множителем при $\eta$ в последнем интеграле (обозначим его через $v(z))$, при $|z| \leqslant 1$ удовлетворяет условиям $0 \leqslant v(z) \leqslant$ $\ln (n+1)$, а при $|z|>1$ - условиям $k \ln |z|^{2}<v(z)<k \ln |z|^{2}+\ln (n+1)$. Следовательно, для рассматриваемых дивизоров значение дефекта (как неванлинновского, так и валироновского) равно $(4 \pi)^{-1} \int_{|z|>1} \eta \ln |z|^{2}$. Если через $U$ обозначить унитарное преобразование пространства $Q$, переводящее точку $q_{0}$ в любую заданную точку $q_{1}$, то для последовательности кривых $g_{k}=U \circ f_{k}$ проходят все предыдущие рассуждения и дефектными окажутся все дивизоры, проходящие через точку $q_{1}$.

ПримеР 3. Для любой последовательности точек $\left\{q_{k}\right\}$ мы построим последовательность кривых, для которых дефектным (с тем же значением дефекта, что и в примере 2) будет любой дивизор, проходящий через какую-нибудь из точек заданной последовательности. Для этого построим последовательность унитарных преобразований $\left\{U_{k}\right\}$ пространства $Q$ так, чтобы $U_{k} q_{0}$ равнялось $q_{k}$, и такую последовательность $\left\{s_{k}\right\}$ натуральных чисел, что каждое натуральное число встречается в ней бесконечное число раз. Положив $g_{k}=U_{l} \circ f_{k}$, где $l$ выбирается равным $s_{k}$, мы, согласно замечанию в конце предыдущего примера, получим нужную последовательность кривых.

Для дальнейшего уточнения структуры множества дефектных значений удобно использовать введенные и исследованные автором в работах $[3,4]$ понятия div-потенциала и div-емкости.

ОПРедЕЛЕниЕ 2. Пусть $\sigma$ - борелевская мера на $M$. Назовем div-nотенииалом этой меры функцию $U_{\text {div }}^{\sigma}$ на $Q$, равную интегралу по мере $\mu$ на множествах $S_{q}$ от потенциала на $M$ меры $\sigma$ относительно ядра $\gamma(y, a)$,

$$
U_{\mathrm{div}}^{\sigma}(q)=\int_{S_{q}}\left(\int_{M} \gamma(y, a) \sigma(d a)\right) \mu=\int_{M}\left(\int_{S_{q}} \gamma(y, a) \mu\right) \sigma(d a) .
$$

ОПРЕДЕЛЕНИЕ 3. div-емкостью компактного множества $E \subset M$ назовем величину $\operatorname{Cap}_{\operatorname{div}} E=\sup \left(1 / \sup U_{\operatorname{div}}^{\sigma}(q)\right)$, где внутренний супремум берется по $q \in Q$, а внешний - по всем вероятностным мерам, носитель которых содержится в $E$.

Понятия внутренней и внешней div-емкостей определяются естественным образом. 
ЗАмЕЧАнИЕ. В условиях примера 1 div-емкость на $M$ лишь постоянным множителем отличается от введенной Мользоном, Шиффманом и Сибони [8] проективной логарифмической емкости. Однако если $Q$ - подмногообразие в двойственном к $M$ проективном пространстве и гиперплоскость $G \subset M$ полярна к точке, не принадлежащей многообразию $Q$, то $G$ имеет положительную div-емкость, в то время как проективная логарифмическая емкость любой гиперплоскости равна нулю.

Для div-емкостей верны многие утверждения классической теории потенциала. В частности, верна следующая лемма.

Лемма 1. Пусть для множества $E$ при любом $\varepsilon>0$ найдется такая последовательность компактов $E_{1}, E_{2}, \ldots$, что $\sum \operatorname{Cap}_{\mathrm{div}} E_{i} \leqslant \varepsilon u E \subset \bigcup E_{i}$. Тогда внешняя div-емкость множества $E$ равна нулю.

Tеорема 4. Пусть компактное множество $B_{0} \subset M$ состоит из таких точек $а$, что норма функиионала $T(f)-N(f, a)$ для этих а больше, чем А. Тогда $\operatorname{div-емкость~} \operatorname{Cap}_{\operatorname{div}} B$ этого множества не превосходит величинь 1/A. Эта же оченка верна и для множества $B$ тех точек, для которых выполняется неравенство $m_{\eta}(f, a)=\int_{\mathbb{C}} f^{*} \zeta_{a} \eta>A$.

ДокАЗАтЕЛЬство. Как и в теореме 2, достаточно доказать только второе утверждение. Проинтегрируем неравенство, задающее множество $B$, по любой вероятностной мере $\sigma$, носитель которой содержится в множестве $B$, воспользуемся определением функций $\zeta_{a}$ и $U_{\text {div }}^{\sigma}$ и поменяем порядок интегрирования. Получим следующие соотношения:

$$
A \leqslant \int_{M}\left(\int_{\mathbb{C}}\left(\int_{S_{f(z)}} \gamma_{a}(y) \mu\right) \eta\right) \sigma(d a) \leqslant \int_{\mathbb{C}} \eta f^{*} U_{\operatorname{div}}^{\sigma} \leqslant \sup _{q} U_{\operatorname{div}}^{\sigma}(q) \int_{\mathbb{C}} \eta .
$$

Последняя величина не превосходит $1 / \mathrm{Cap}_{\operatorname{div}} B$.

Теорема 5. Пусть $\left\{f_{k}\right\}$ - последовательность кривьх $u\left\{d_{k}\right\}$ - последовательность их степеней. Если ряд $\sum d_{k}^{-1}$ сходится, то множество $\Delta$ значений параметров, соответствуюших дивизорам, дефектньм по Валирону, имеет div-емкость, равную нулю.

ДокАЗАтЕЛЬство. Построим такую монотонно стремящуюся к бесконечности последовательность положительных чисел $\left\{s_{k}\right\}$, что ряд $\sum\left(s_{k} / d_{k}\right)$ все еще будет сходиться. Обозначим через $B_{k}$ множество тех значений $a \in M$, для которых выполняется неравенство $\int_{\mathbb{C}} \zeta_{a}\left(f_{k}(z)\right) \eta>d_{k} / s_{k}$. Согласно теореме 4 , div-емкость этого множества не превосходит величины $s_{k} / d_{k}$. С другой стороны, каждое $a$, соответствующее дефектному дивизору, содержится в бесконечной подпоследовательности множеств $B_{k}$, т. е. для любого натурального $j$ выполнено включение $\Delta \subset \bigcup_{k>j} B_{k}$. Утверждение теоремы следует из леммы 1 , если учесть сходимость ряда $\sum\left(s_{k} / d_{k}\right)$.

В оставшейся части работы мы более подробно исследуем ситуацию, когда $M$ параметризует линейную систему дивизоров на многообразии $Q$, т.е. когда на $Q$ задано голоморфное расслоение $L$ на комплексные прямые и $M$ - результат проективизации некоторого линейного пространства $M_{1}$ сечений этого расслоения. Предполагается, что у $M_{1}$ отсутствуют базисные точки, т. е. точки, в которых обращаются в нуль одновременно все сечения пространства $M_{1}$. Рассмотрим отображение расслоений $Q \times M_{1} \rightarrow L$, переводящее пару 
$(q, y) \in Q \times M_{1}$ в значение сечения $y$ в точке $q$. Обозначим через $W_{1}$ расслоение, являющееся ядром этого отображения, а через $W$ - результат послойной проективизации расслоения $W_{1}$. Многообразие $W$, которое можно считать вложенным в прямое произведение $Q \times M$, удовлетворяет всем нужным требованиям.

Выберем в пространстве $M_{1}$ базис $\beta_{0}, \beta_{1}, \ldots, \beta_{m}$, который мы будем считать ортонормированным, и в качестве однородных координат в $M$ возьмем коэффициенты разложения $y=y_{0} \beta_{0}+\cdots+y_{m} \beta_{m}$. Формы $\lambda, \mu$ и $\omega$, а также функция $\gamma_{a}(y)$ для рассматриваемого случая построены в примере 1. Для вычисления функции $\zeta_{a}(q)$, являющейся div-потенциалом атомарной меры с носителем в точке $a \in M$, обозначим через $w(q)$ точку из $M$, соответствующую дивизору на $Q$, определяемому сечением, ортогональным ко всем сечениям, обращающимся в нуль в точке $q$, и построим на $M$ функцию $\xi(a, w)$, равную интегралу от формы $\gamma(a, y) \mu$ по $y$, где $y$ пробегает гиперплоскость, полярную точке $w$. Таким образом, $\zeta_{a}(q)=\xi(a, w(q))$. Функция $\gamma_{a}(y)$ симметрична по переменным $a$ и $y$, и потому по $a$ она удовлетворяет тому же уравнению $\left.d_{a} d_{a}^{c}\left(\gamma(a, y) \mu_{a}\right)\right)=\omega_{a}$, что и по $y$. Умножив обе части этого уравнения на $\mu_{y}$ и проинтегрировав по гиперплоскости, полярной точке $w$, получим $\left.d_{a} d_{a}^{c}\left(\xi(a, w) \mu_{a}\right)\right)=(m !)^{(m-1) / m} \omega_{a}$. Из унитарной инвариантности функции $\gamma(a, y)$ и формы $\mu$ следует, что этим же свойством обладает и функция $\xi(a, w)$, а это означает, что она зависит только от расстояния между точками $a$ и $w$, равного модулю угла между векторами, задающими эти точки. Удобнее пользоваться переменной $t$, которая является квадратом тангенса этой величины и в однородных координатах задается формулой $t=\left(\langle a, a\rangle\langle w, w\rangle-|\langle a, w\rangle|^{2}\right) /|\langle a, w\rangle|^{2}$. Подставляя в приведенное выше уравнение вместо $\xi(a, w)$ функцию $h(t)$, получим для нее уравнение

$$
t h^{\prime \prime}+\frac{m+t}{1+t} h^{\prime}=\frac{m}{4 \pi} \frac{1}{(1+t)^{2}} .
$$

Единственным его решением, не имеющим особенностей при $t=0$ и удовлетворяющим условию $h(0)=0$, является функция $h(t)=(4 \pi)^{-1} \ln (1+t)$, и потому для $\zeta_{a}(q)$ в однородных координатах на многообразии $M$ получаем $\zeta_{a}(q)=(4 \pi)^{-1} \ln (\langle a, a\rangle\langle w(q), w(q)\rangle) /|\langle a, w(q)\rangle|^{2}$.

Пусть теперь $\sigma$ - вероятностная мера на $s$-мерном линейном многообразии $S$ пространства $M$, определяемая соответствующим образом нормированным ограничением на $S$ метрики Фубини-Штуди. Среднее по $a \in S$ значение функции $\zeta_{a}(q)$ совпадает с $\operatorname{div}$-потенциалом $U_{\mathrm{div}}^{\sigma}(q)$ меры $\sigma$. Этим мотивируется введение следующего определения.

ОПределЕние 4. Пусть $S$ - линейное многообразие в $M$ и $\sigma$ - описанная выше мера на $S$. Для кривой $f$ величину $\int_{\mathbb{C}} U_{\text {div }}^{\sigma}(f(z)) \eta$ обозначим через $m_{\eta}(f, S)$, а для последовательности кривых $f_{1}, \ldots, f_{k}, \ldots$ со степенями $d_{1}, \ldots$, $d_{k}, \ldots$ величину $\liminf _{k \rightarrow \infty}\left(m_{\eta}\left(f_{k}, S\right) / d_{k}\right)$ назовем усредненнылм неванлинновским дефектом и обозначим через $\delta_{N}(S)$. Величину $\lim \sup _{k \rightarrow \infty}\left(m_{\eta}\left(f_{k}, S\right) / d_{k}\right)$ назовем усредненным валироновским дефектом и обозначим через $\delta_{V}(S)$.

Мы хотим изучить усредненные значения дефектов в сопоставлении с их индивидуальными значениями. Фундаментальную роль при этом будет играть следующая лемма.

Лемма 2. Для линейного многообразия $S$ и описанной выше меры $\sigma$ на нем существует такая константа с, зависящая только от размерности s многообразия $S$, что при всех $q \in Q$ выполнено соотношение $U_{\operatorname{div}}^{\sigma}(q)=\inf _{a \in S} \zeta_{a}(q)+c$. 
ДокАЗАТЕЛЬСтво. Если точка а принадлежит гиперплоскости, полярной точке $w(q)$, то $\zeta_{a}(q)$ обращается в бесконечность, и, значит, в случае когда $S$ содержится в этой гиперплоскости, утверждение леммы становится тривиальным. Поэтому мы ограничимся евклидовой частью пространства $M$ с координатами $\left(\alpha_{1}, \ldots, \alpha_{n}\right)$ и будем считать, что точка $w(q)$ находится в начале координат, а многообразие $S$ задается уравнениями $\alpha_{s+1}=$ const, $\ldots, \alpha_{n}=$ const. Обозначим через $r_{0}$ евклидово расстояние от начала координат до многообразия $S$, а через $\rho$ - расстояние от начала до точки $a$. В этих обозначениях функция $\zeta_{a}(q)$ записывается как $(4 \pi)^{-1} \ln \left(1+\rho^{2}\right)$, а мера $\sigma$ задается формой $\left(\frac{1}{4 \pi} d d^{c} \ln \left(1+r_{0}^{2}+\left|\alpha_{1}\right|^{2}+\cdots+\left|\alpha_{s}\right|^{2}\right)\right)^{s}=\left(\frac{1}{4 \pi} d d^{c} \ln \left(1+\frac{\left|\alpha_{1}\right|^{2}}{1+r_{0}^{2}}+\cdots+\frac{\left|\alpha_{s}\right|^{2}}{1+r_{0}^{2}}\right)\right)^{s}$.

Определим величину $V(r)$ как меру той части многообразия $S$, которая находится вне шара радиуса $r$ с центром в начале координат, и введем обозначения $\beta_{i}=\alpha_{i} / \sqrt{1+r_{0}^{2}},|\beta|^{2}=\left|\beta_{1}\right|^{2}+\cdots+\left|\beta_{s}\right|^{2}$ и $r_{1}=\left(r^{2}-r_{0}^{2}\right) /\left(1+r_{0}^{2}\right)$. Тогда

$$
V(r)=\int_{|\beta|^{2} \geqslant r_{1}}\left(\frac{1}{4 \pi} d d^{c} \ln \left(1+|\beta|^{2}\right)\right)^{s}=\frac{s !}{\pi^{s}} \int_{|\beta|^{2} \geqslant r_{1}} \frac{d v_{\text {евкл }}}{\left(1+|\beta|^{2}\right)^{s+1}} .
$$

Последний интеграл нетрудно вычислить, перейдя к полярным координатам. Он равен

$$
s \int_{r_{1}}^{\infty} \frac{\rho^{s-1}}{(1+\rho)^{s+1}} d \rho=s \sum_{k=0}^{s-1} \frac{(-1)^{k}}{k+1} C_{s-1}^{k}\left(\frac{1+r_{0}^{2}}{1+r^{2}}\right)^{k+1} .
$$

В частности, $V\left(r_{0}\right)=1$.

Для $\operatorname{div}$-потенциала $U_{\mathrm{div}}^{\sigma}(q)$ теперь имеем

$$
\begin{aligned}
U_{\text {div }}^{\sigma}(q) & =\int_{S} \zeta(a, q) \sigma(d a)=-\frac{1}{4 \pi} \int_{r_{0}}^{\infty} \ln \left(1+\rho^{2}\right) d V(\rho) \\
& =\frac{1}{4 \pi} \ln \left(1+r_{0}^{2}\right)+\frac{1}{2 \pi} \int_{r_{0}}^{\infty} \frac{V(\rho)}{1+\rho^{2}} \rho d \rho \\
& =\frac{1}{4 \pi} \ln \left(1+r_{0}^{2}\right)+\frac{s}{4 \pi} \sum_{k=0}^{s-1}(-1)^{k} \frac{C_{s-1}^{k}}{(k+1)^{2}},
\end{aligned}
$$

что доказывает лемму.

Из полученной оценки непосредственно следует

Теорема 6. Если в описанной ситуаиии последовательность кривых такова, что последовательность их степеней стремится $\kappa$ бесконечности, то при любом $a \in S$ выполняются неравенства

$$
\delta_{N}(a) \geqslant \delta_{N}(S), \quad \delta_{V}(a) \geqslant \delta_{V}(S) .
$$

Что касается обратных неравенств, то, как мы сейчас увидим, они выполняются лишь для почти всех $a \in S$ и при этом в случае валироновских дефектов требуются дополнительные условия на рост последовательности степеней.

Tеорема 7. B той же ситуаиии, что и в теореме 6 , для всех $a \in S$, за исключением принадлежаших некоторому множеству, имеющему $\sigma$-меру нуль, выполняется неравенство $\delta_{N}(a) \leqslant \delta_{N}(S)$. Если же последовательность кривых такова, что ряд из чисел, обратных их степеням, сходится, то для всех 
$a \in S$, за исключением принадлежаших некоторому множеству, имеюшему $\sigma$-меру нуль, вьполняется неравенство $\delta_{V}(a) \leqslant \delta_{V}(S)$.

ДокАЗАтЕЛьство. При доказательстве первого утверждения, переходя, если нужно, к подпоследовательности, можно считать, что $\lim _{k \rightarrow \infty}\left(m_{\eta}\left(f_{k}, S\right) / d_{k}\right)$ $=\delta_{N}(S)$. Тогда из леммы 2 следует, что для некоторой стремящейся к нулю последовательности $\varepsilon_{1}, \ldots, \varepsilon_{k}, \ldots$ функции $g_{k}(a)=m_{\eta}\left(f_{k}, a\right) / d_{k}-\delta_{N}(S)+\varepsilon_{k}$ положительны при всех $a \in M$. С другой стороны, $\int_{S} g_{k}(a) \sigma(d a)=m_{\eta}\left(f_{k}, S\right) / d_{k}-$ $\delta_{N}(S)+\varepsilon_{k}$. Правая часть при $k \rightarrow \infty$ стремится к нулю. Мы получили последовательность положительных функций, интегралы от которых стремятся к нулю, сходящуюся, следовательно, к нулю по мере. Из нее можно выделить подпоследовательность, сходящуюся к нулю почти всюду. Взяв нижний предел (уже по всей последовательности), получаем первое утверждение теоремы.

Чтобы доказать теорему для валироновских дефектов, обозначим через $m_{S}(f, a)$ величину $m_{\eta}(f, a)-m_{\eta}(f, S)$ и оценим $\sigma$-меру множества $W^{L}(f)=$ $\left\{a \in S: m_{S}(f, a) \geqslant L\right\}$. Из определения следует, что интеграл по всему $S$ от функции $m_{S}(f, a)$ равен нулю, в то время как из неравенства $\zeta_{a}(q)-U_{\operatorname{div}}^{\sigma}(q) \geqslant-c$ (лемма 2) следует, что интеграл от этой функции по дополнению к множеству $W^{L}(f)$ больше либо равен $-c$. А это значит, что $\int_{W^{L}(f)} m_{S}(f, a) \sigma(d a) \leqslant c$, т. е. $\sigma$-мера множества $W^{L}(f)$ не превосходит величины $\delta / L$.

Построим теперь, как и в доказательстве теоремы 5 , такую монотонно стремящуюся к бесконечности последовательность положительных чисел $\left\{s_{k}\right\}$, что ряд $\sum\left(s_{k} / d_{k}\right)$ все еще сходится. Пусть для некоторого значения а справедливо неравенство $\delta_{V}(a)>\delta_{V}(S)$. Выберем такую подпоследовательность индексов, что при стремлении $k$ к бесконечности по этой подпоследовательности выполняется соотношение $\lim \left(m_{\eta}\left(f_{k}, a\right) / d_{k}\right)=\delta_{V}(a)$. Тогда для этого значения $a$ при всех достаточно больших значениях $k$ имеет место неравенство $m_{\eta}\left(f_{k}, a\right)-m_{\eta}\left(f_{k}, S\right)>d_{k} / s_{k}$, т. е. каждое такое $a$ при всех $j$ принадлежит множеству $\bigcup_{k>j} W^{d_{k} / s_{k}}\left(f_{k}\right)$, что с учетом оценки меры множества $W^{d_{k} / s_{k}}\left(f_{k}\right)$ доказывает нужное утверждение.

\section{ЛИТЕРАТУРА}

1. Дектярев И. М. Усредненные дефекты голоморфных отображений. В кн.: Многомерный комплексный анализ, Изд-во Ин-та физики СО АН СССР, Красноярск, 1985 , с. $64-77$.

2. Дектярев И. М. Равнораспределенность значений при полиномиальных отображениях. Функц. анализ и его прил., 33, вып. 4, 70-73 (1999).

3. Дектярев И. М. Замечание о структуре множества дефектных дивизоров. ДАН CCCP, 292, № 4, 800-803 (1987).

4. Дектярев И. М. Емкость и дефектные дивизоры. Функц. анализ и его прил., 22, вып. 1, 64-65 (1988).

5. Гриффитс Ф., Харрис Дж. Принципы алгебраической геометрии. Мир, М., 1982.

6. Ландкоф Н. С. Основы современной теории потенциала. Наука, М., 1966.

7. Russakovskii A., Sodin M. Equidistribution for sequences of polynomial mappings. Indiana Univ. Math. J., 44, No. 3, 851-882 (1995).

8. Molzon R., Shiffman B., Sibony N. Average growth estimates for hyperplane sections of entire analytic sets. Math. Ann., 257, 43-59 (1981). 